\section{Estudio transversal de prevalencia y características del dolor crónico en pacientes con esquizofrenia}

\author{
MAGALY SEPÚLVEDA-TORRES ${ }^{1, \mathrm{a}}$, \\ CRISTHIAN CARVAJAL ${ }^{2}$, ÁLVARO CAVIERES F. ${ }^{3,4}$
}

\section{Prevalence and features of chronic pain among patients with schizophrenia}

\begin{abstract}
Background: People with schizophrenia generally refrain from manifesting somatic symptoms, and may not complain of pain. Therefore these patients may have an insensitivity to pain. Aim: To determine the prevalence of chronic pain in a sample of patients with schizophrenia. Patients and Methods: The McGill Pain Questionnaire (MPQ) was answered by 79 outpatients with schizophrenia with a mean age of 47 years (67\% men) attended at a public hospital in Chile. Results: Chronic pain, defined as lasting more than six months, was reported by $15.2 \%$ of patients. Acute pain was reported by $63 \%$ of patients. No significant differences were observed in sociodemographic characteristics or duration of illness between patients with acute or chronic pain. The assessment of the different pain dimensions showed a predominance of sensory and affective components, with pain rating indexes of 0.82 and 0.71 , respectively. Conclusions: These results indicate that the prevalence of pain among people with schizophrenia, is similar to that of the general population.
\end{abstract}

(Rev Med Chile 2021; 149: 1023-1030)

Key words: Chronic Pain; Pain Measurement; Schizophrenia.

'Escuela de Medicina, Universidad
de Valparaíso, San Felipe, Chile.
'Escuela de Medicina, Universidad
de Valparaíso. Viña del Mar,
Chile.
${ }^{3}$ Departamento de Psiquiatría,
Escuela de Medicina, Universidad
de Valparaíso. Valparaíso, Chile.
${ }^{4}$ Hospital del Salvador.
Valparaíso, Chile.
aMsC.
Trabajo no recibió
financiamiento.
Los autores declaran no tener
conflictos de interés.
Recibido el 26 de mayo de 2020,
aceptado el 10 de mayo de 2021.
Correspondencia a:
Magaly Sepúlveda Torres
Los Crisantemos 2160,
Departamento 501, Bloque B.
San Felipe. Chile.
magaly.sepulveda@postgrado.
uv.cl

S egún la Asociación Internacional para el Estudio del Dolor (IASP), este se define como "una experiencia sensorial y emocional desagradable asociada con daño tisular real o potencial, o descrito en términos de tal daño". La incapacidad de comunicarse no excluye la posibilidad de que una persona lo sufra y requiera un tratamiento para aliviarlo ${ }^{1}$. Esto es aplicable a los trastornos mentales y médicos que ocasionan impedimentos en la comunicación y subraya la necesidad de explorar atentamente la expresión del dolor.

La experiencia subjetiva del dolor resulta de la integración de tres aspectos; sensoriales, principalmente su intensidad, calidad y ubicación; motivacionales-afectivo, o las sensaciones desagradables, miedo, ansiedad, y la activación neurovegetativa que acompaña a los estímulos dolorosos; y cognitivo-evaluativos, incluyendo procesos como la memoria ${ }^{2}$. Desde un punto de vista neurobiológico, el procesamiento del dolor depende de dos vías neurales; el sistema anterolateral, que incluye el tálamo y la corteza parietal, encargado del aspecto sensorial-discriminativo ${ }^{3}$; y el sistema medial, vinculado a aspectos motivacionales-afectivos y cognitivos, que comprende las proyecciones de los tractos espinotalámico, espinomesencefálico y espinoreticular a la amígdala, corteza cingulada anterior, núcleos hipotalámicos e hipocampo ${ }^{4}$. El procesamiento final de la información ocurre en la corteza prefrontal, tálamo e hipocampo, mientras que la corteza cingulada anterior (sistema de dolor medial), además de cumplir un rol en el control atencional ${ }^{5}$, ejerce acciones de inhibición sobre 
la sustancia gris periacueductal -vinculada a la supresión del dolor ${ }^{6}$.

La esquizofrenia, tiene una prevalencia mundial es de $0,46 \%{ }^{7}$. Además de los síntomas psicóticos y deficitarios ${ }^{8}$, Kraepelin $^{9}$, relata que los pacientes podían quemarse con cigarrillos y experimentar pinchazos con agujas o lesionarse sin mostrar reacciones adaptativas y normales. Por su parte, Bleuler ${ }^{10}$ realizó observaciones similares sobre la disminución de la reactividad a estímulos dolorosos en el cuerpo o la piel de los pacientes.

A lo largo del tiempo el reporte de un número significativo de casos de personas con esquizofrenia y enfermedades médicas dolorosas (abdomen agudo, apendicitis, peritonitis, úlcera péptica, intestino perforado, síndrome compartimental, fracturas) con escaso o ningún reporte de dolor, contribuyó al concepto de la insensibilidad al dolor en la esquizofrenia ${ }^{11}$.

Más recientemente, un metaanálisis de 11 estudios con controles voluntarios sanos, sometidos a procedimientos experimentales para inducir el dolor (por ejemplo, estímulos térmicos, eléctricos o mecánicos) confirma una respuesta disminuida al dolor inducido experimentalmente, incluyendo a pacientes sin tratamiento antipsicótico, quienes además presentan umbrales sensoriales aumentados respecto del grupo control ${ }^{12}$.

Un análisis similar, pero en relación con el dolor producido naturalmente, concluye que este tipo de dolor afecta a un tercio de las personas con esquizofrenia, con intensidades similares a los grupos de control ${ }^{13}$. Un estudio posterior, concluyó que, tanto la prevalencia como la intensidad del dolor, parecen estar disminuidas en las personas con esquizofrenia, pero solo cuando el dolor es producto de una intervención médica. En otro tipo de situaciones, la prevalencia y la intensidad del dolor son comparables a los controles $^{14}$.

Se han postulado algunas hipótesis para explicar la hipoalgesia en las personas con esquizofrenia. Aunque la neuropatología no está del todo clara $^{2}$, numerosos estudios muestran cambios patológicos en las áreas cerebrales implicadas en el procesamiento del dolor ${ }^{3}$. Los hallazgos más robustos se han encontrado en la corteza prefrontal y en el hipocampo ${ }^{2}$, lo que sugiere una potencial alteración de los aspectos motivacionales-afectivos y cognitivo-evaluativos del dolor.

Clínicamente, la disminución de la sensibili- dad al dolor se ha relacionado con los síntomas psicóticos, el aplanamiento afectivo y/o déficit de atención ${ }^{15}$. Además, se ha observado hipoalgesia en pacientes medicados y libres de fármacos, lo que sugiere que la administración de antipsicóticos no estaría relacionada con el fenómeno ${ }^{16}$. Otra posibilidad es que los pacientes se abstienen de manifestar el dolor para evitar ser una carga y por el temor de ser hospitalizados ${ }^{17}$.

Lo anterior es preocupante, ya que las personas con esquizofrenia tienen más probabilidades de experimentar una variedad de comorbilidades médicas que pueden inducir dolor ${ }^{18}$. El hecho de que los pacientes no reconozcan o no refieran el dolor, puede contribuir a las barreras en la atención o a ocultar la necesidad de tratamiento médico ${ }^{12}$. Por ejemplo, a pesar de las altas tasas de alteraciones cardiometabólicas en pacientes con esquizofrenia, pocos pacientes solicitan atención por esta causa, lo que puede contribuir a una mortalidad prematura por causas médicas ${ }^{13}$.

La prevalencia de dolor crónico (mayor a seis meses) en pacientes con esquizofrenia en diferentes estudios en el mundo muestra una amplia variación, que va de $8 \%$ a $74 \%$, dependiendo de los diseños y métodos de recolección de dato ${ }^{19,20,21}$. En Chile no hay información acerca de la prevalencia del dolor en pacientes con patologías psiquiátricas, mientras que, en la población general, se estima en $41,1 \%$.

Dado que los estudios internacionales publicados varían tanto por la metodología utilizada, como por factores relacionados con la recolección de los datos ${ }^{23}$, es importante estimar la prevalencia de dolor crónico en una muestra de pacientes con esquizofrenia de nuestro medio, como asimismo analizar su relación con variables clínicas y sociodemográfica y caracterizar el dolor principal presentado por los sujetos en cuanto a: localización, frecuencia, tiempo, intensidad, factores agravantes y aliviadores del dolor.

Reportamos a continuación los datos obtenidos aplicando el McGill Pain Questionnaire $(\mathrm{MPQ})^{24}$ a una muestra de personas con esquizofrenia en control ambulatorio en el Policlínico del Hospital del Salvador de Valparaíso. El MPQ evalúa no solo la intensidad del dolor sino también sus cualidades afectivas y motivadoras del dolor. Aunque el tiempo requerido para que el sujeto complete correctamente el cuestionario puede ser una limitante para el empleo rutinario de esta 
herramienta, existe amplia evidencia de su confiabilidad y validez para cuantificar la experiencia del dolor consciente ${ }^{25}$.

\section{Pacientes y Método}

Estudio de corte transversal de prevalencia en muestra probabilística. Para el cálculo del tamaño de la muestra se utilizó el programa Granmo, considerando los siguientes parámetros: prevalencia de dolor crónico en los pacientes psiquiátricos $25 \%{ }^{20,21,23}$, un error de $5 \%$ y un intervalo de confianza de $95 \%$ para una muestra de 79 sujetos.

Los criterios de inclusión en la muestra fueron: 18-55 años, diagnóstico de esquizofrenia, realizado por médico psiquiatra, según $(\mathrm{CIE}-10)^{26}$, en tratamiento ambulatorio durante, al menos dos años en el Policlínico del Hospital Del Salvador de Valparaíso y estables en su condición clínica, escolaridad mínima de ocho años y poseer habilidades de comprensión y verbalización para responder los cuestionarios. No se incluyeron personas con discapacidad intelectual (verificado en la evaluación médica y revisión de la ficha clínica) o con habla o comportamiento desorganizado, desorientación auto o alopsíquica, agresividad o irritabilidad, o patologías médicas o neurológicas severas o que produzcan alteración de la percepción del dolor (ej. neuropatía diabética, neuralgias, enfermedades reumatológicas y musculoesqueléticas).

El protocolo del estudio fue autorizado por el Comité de Evaluación Ética-Científica del Servicio de Salud Valparaíso-San Antonio. Todos los participantes entregaron su consentimiento informado por escrito antes de completar el cuestionario.

El McGill Pain Questionnaire (MPQ) ${ }^{27}$ evalúa la intensidad del dolor y sus cualidades afectivas y motivacionales. Incluye un dibujo anatómico de la forma humana para ubicar el dolor y una escala de descripción verbal (VDS) que permite registrar el nivel de intensidad de la experiencia dolorosa actual, además de un inventario de 72 descriptores (10 grupos sensoriales, 5 grupos afectivos, 1 evaluativo y 4 misceláneos). El paciente debe revisar esta lista y marcar con un círculo los que mejor describen su experiencia actual de dolor. Cada dimensión del MPQ se puntúa individualmente y también se registra una puntuación total acumulativa. El cociente entre los grupos de las distintas áreas (sensorial, afectivo, cognitivo y misceláneas) y el total de adjetivos descritos da como resultado el PRI (Índice de valoración del dolor). Aunque el tiempo requerido para completar el MPQ limita su empleo (15 a 20 minutos), se ha demostrado su confiabilidad y validez para cuantificar la experiencia del dolor ${ }^{27}$.

El instrumento McGill se evaluó en un box individual, en el que los pacientes realizaban un autorreporte y un investigador estaba presente solo para eventuales dudas, sin interferir en el desarrollo de este. Se realizó entre los meses de agosto de 2019 y marzo de 2020.

\section{Análisis estadístico}

Para el análisis de los datos se utilizó el programa estadístico GraphPad 6.0 para MacOS y se analizaron utilizando la estadística descriptiva. Se calculó la prevalencia de síntomas dolorosos entre los participantes con esquizofrenia, con un intervalo de confianza de 95\%. La asociación entre el desenlace y las variables independientes fueron analizadas por medio de la prueba Chi-cuadrado de Pearson y para comparar las variables continuas se utilizó la prueba t de Student; considerando un $\alpha=5 \%$.

\section{Resultados}

La muestra estuvo constituida mayoritariamente por hombres $(66,6 \%)$, con un promedio de edad de 47,21 años. Ninguno de los encuestados tenía pareja, su escolaridad promedio alcanzó hasta el segundo ciclo básico $(66,6 \%)$, la mitad no realizaba labores fuera de casa $(50 \%)$ y el tiempo de enfermedad fue en promedio 13,5 meses (Tabla 1).

Del total de pacientes evaluados (79), 12 $(15,2 \%)$ reportaron dolor crónico, definido como una duración mayor a 6 meses; y $32(62,5 \%)$ pacientes dolor agudo, es decir, con duración menor a 6 meses. Al comparar ambos grupos, no se observaron diferencias significativas en las variables sociodemográfica y tiempo de enfermedad (Tabla 1). El análisis de índice de valoración del dolor mostró un predominio del componente sensorial $(\mathrm{PRI}=0,82)$, seguido por el componente afectivo (PRI $=0,71)$ (Figura 1$)$.

El dolor más frecuentemente reportado fue en cabeza/cara/boca (33,3\%), seguido de las ubicaciones lumbar/sacra/coccígea (16,6\%) y miembros 
Tabla 1. Características sociodemográficas y tiempo de enfermedad según tipo de dolor (agudo vs crónico)

\begin{tabular}{|c|c|c|c|c|c|c|c|}
\hline \multirow[t]{2}{*}{ Variables } & \multicolumn{3}{|c|}{$\begin{array}{l}\text { Dolor menor de } 6 \text { meses } \\
\qquad(n=20)\end{array}$} & \multicolumn{3}{|c|}{$\begin{array}{l}\text { Dolor mayor de } 6 \text { meses } \\
\qquad(n=12)\end{array}$} & \multirow[b]{2}{*}{$p$} \\
\hline & $\mathrm{n}$ & $\%$ & Media & $\mathrm{n}$ & $\%$ & Media & \\
\hline $\begin{array}{l}\text { Género } \\
\text { Femenino } \\
\text { Masculino }\end{array}$ & $\begin{array}{r}7 \\
13\end{array}$ & $\begin{array}{l}35,00 \\
65,00\end{array}$ & & $\begin{array}{l}4 \\
8\end{array}$ & $\begin{array}{l}33,33 \\
66,66\end{array}$ & & 0,92 \\
\hline 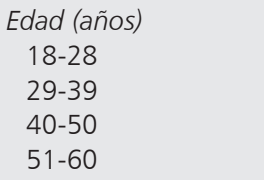 & $\begin{array}{l}2 \\
4 \\
9 \\
5\end{array}$ & $\begin{array}{l}10,00 \\
20,00 \\
45,00 \\
25,00\end{array}$ & 42,82 & $\begin{array}{l}1 \\
2 \\
7 \\
2\end{array}$ & $\begin{array}{l}8,33 \\
16,67 \\
58,33 \\
16,6\end{array}$ & 47,2 & 0,28 \\
\hline $\begin{array}{l}\text { Estado civil } \\
\text { Soltero } \\
\text { Divorciado }\end{array}$ & $\begin{array}{r}19 \\
1\end{array}$ & $\begin{array}{r}95,00 \\
5,00\end{array}$ & & 12 & 100,00 & & 0,43 \\
\hline $\begin{array}{l}\text { Escolaridad } \\
1^{\circ} \text { ciclo básico } \\
2^{\circ} \text { ciclo básico } \\
\text { Enseñanza media } \\
\text { Enseñanza superior }\end{array}$ & $\begin{array}{r}5 \\
10 \\
3 \\
2\end{array}$ & $\begin{array}{l}25,00 \\
50,00 \\
15,00 \\
10,00\end{array}$ & & $\begin{array}{l}3 \\
8\end{array}$ & $\begin{array}{r}25,00 \\
66,67 \\
8,33\end{array}$ & & 0,85 \\
\hline $\begin{array}{l}\text { Ocupación } \\
\text { Labores de casa } \\
\text { Estudiante } \\
\text { Trabajador } \\
\text { Desocupado }\end{array}$ & $\begin{array}{r}12 \\
1 \\
4 \\
3\end{array}$ & $\begin{array}{r}60,00 \\
5,00 \\
20,00 \\
15,00\end{array}$ & & $\begin{array}{l}6 \\
4\end{array}$ & $\begin{array}{l}50,00 \\
33,33 \\
16,66\end{array}$ & & 0,73 \\
\hline $\begin{array}{l}\text { Años de enfermedad } \\
\begin{array}{l}1-5 \\
5-10 \\
10-15 \\
15-20 \\
200>\end{array}\end{array}$ & $\begin{array}{l}2 \\
4 \\
6 \\
2 \\
6\end{array}$ & & & $\begin{array}{l}1 \\
3 \\
2 \\
1 \\
5\end{array}$ & $\begin{array}{r}8,33 \\
25,00 \\
16,67 \\
8,33 \\
41,67\end{array}$ & & 0,15 \\
\hline
\end{tabular}

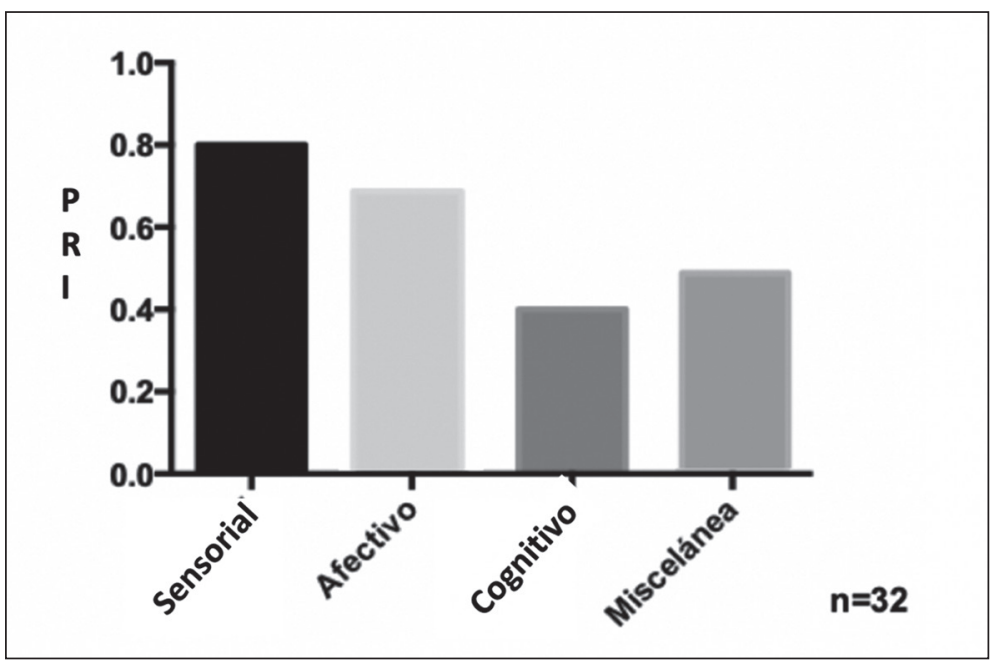
dolor agudo y crónico en pacientes con esquizofrenia. 
inferiores (16,6\%). De los encuestados, 33,3\% informó dolor una vez por semana, seguido por $25 \%$ cada 2 semanas. El tiempo promedio de dolor fue de 22 meses. La variación de la intensidad del dolor en la última semana mostró que el dolor más frecuente fue leve (75\%), seguido de horrible $(16,6 \%)$. Con relación al uso de analgésicos $66,67 \%$ indicó que los utiliza. Los factores más indicados como fuente de alivio del dolor fueron: Posición (sentado, bipedastación, decúbito), frío, calor y reposo, mientras que los que lo agravan fueron: Posición, ruido y estrés (Tabla 2).

Tabla 2. Características del dolor crónico reportado por sujetos

\begin{tabular}{|c|c|c|}
\hline Variables & $\mathbf{n}$ & $\%$ \\
\hline \multicolumn{3}{|l|}{ Localización } \\
\hline Abdomen & 1 & 8,33 \\
\hline Cabeza, cara, boca & 4 & 33,33 \\
\hline Lumbar, sacro y coxis & 2 & 16,67 \\
\hline Miembros inferiores & 2 & 16,67 \\
\hline Hombros y miembros superiores & 1 & 8,33 \\
\hline Región cervical & 2 & 15,67 \\
\hline \multicolumn{3}{|l|}{ Frecuencia del dolor principal } \\
\hline 1 vez al día & 2 & 16,67 \\
\hline $2-3$ veces al semana & 4 & 33,33 \\
\hline 1 vez a la semana & 2 & 16,67 \\
\hline Cada 2 semanas & 3 & 25,00 \\
\hline 1 vez al mes & 1 & 8,33 \\
\hline \multicolumn{3}{|l|}{ Uso de analgésicos } \\
\hline Sí & 8 & 66,67 \\
\hline No & 4 & 33,33 \\
\hline \multicolumn{3}{|l|}{ Intensidad del dolor } \\
\hline Ligero & 9 & 75,00 \\
\hline Molesto /Angustioso & 1 & 8,33 \\
\hline Horrible & 2 & 16,67 \\
\hline \multicolumn{3}{|l|}{ Factores que alivian el dolor } \\
\hline Fármacos & 3 & 25,00 \\
\hline Posición (sentado, bipedestación, decúbito) & 2 & 16,67 \\
\hline Cambios de posición & 3 & 25,00 \\
\hline Frío & 1 & 8,33 \\
\hline Calor & 1 & 8,33 \\
\hline Reposo & 2 & 16,67 \\
\hline \multicolumn{3}{|l|}{ Factores que agravan el dolor } \\
\hline Posición (sentado, bipedastación, decúbito) & 4 & 33,33 \\
\hline Frío & 2 & 16,67 \\
\hline Ruido & 4 & 33,33 \\
\hline Estrés & 2 & 16,67 \\
\hline
\end{tabular}

\section{Discusión}

La experiencia del dolor resulta de la integración de aspectos sensoriales, afectivos y cognitivo-evaluativos ${ }^{2}$. Los participantes del estudio reportaron un índice de valoración del dolor con predominio del componente sensorial $(\mathrm{PRI}=0,82)$, seguido por el afectivo $(\mathrm{PRI}=0,71)$ y el cognitivo, considerablemente más bajo $(\mathrm{PRI}=0,43)$ (Figura 1). Esto sugiere una conservación en los mecanismos de dolor lateral (sensorial-discriminativo), y una posible afectación del sistema de dolor medial (emocional-cognitivo).

Una posible interpretación de estos resultados, es que una alteración en la corteza prefrontal dorsolateral (CPFDL), con un rol inhibidor del sistema de dolor medial, en particular de la Corteza del Cíngulo Anterior (CCA), podría causar un incremento del componente afectivo ${ }^{28,29}$. A su vez, la alteración de la CPFDL y de las conexiones entre las áreas de la CPF, altamente relacionado con los déficits cognitivos observados en esquizofrenia $^{30}$, podría dar cuenta de la menor valoración de estos aspectos por parte de los pacientes de nuestro estudio.

Si bien, casi todos los participantes de este estudio describieron adecuadamente su dolor, este buen reporte fue posible gracias al interrogatorio dirigido, y no al reporte espontáneo. Sin embargo, los pacientes consultan infrecuentemente ${ }^{31}$, lo que se suma a la dificultad para describir adecuadamente sus síntomas físicos ${ }^{32}$, falta de tratamiento adecuado $^{33,34}$ y las barreras existentes para su atención ${ }^{35}$.

El dolor crónico fue reportado por 15,2\% de la muestra, un valor dentro del amplio rango de prevalencia de otros estudios; entre $8 \%$ a $74 \%{ }^{13,23}$ para pacientes con trastornos psicóticos y maníacos, y de $20 \%$ a $61 \%$ para la población general ${ }^{22}$. Este resultado es inferior a $36,6 \%$ reportado en Brasil en pacientes con esquizofrenia ${ }^{23}$; mientras que un estudio de cefalea y trastorno bipolar mostró una prevalencia de $48 \%{ }^{20}$ y otro realizado en Londres y Edmonton, informó 18\% de dolor crónico en pacientes con esquizofrenia ${ }^{36}$. Por otro lado, nuestra estimación resultó más alta que $8 \%$ encontrado por Chatuverdi y cols. ${ }^{37}$ en pacientes psicóticos y con trastornos del ánimo de manejo ambulatorios. En cuanto a la prevalencia del dolor crónico en la población adulta general, también existe una gran variación en los resultados obteni- 
dos: $14 \%$ en Escocia $^{38}$, semejando bastante nuestra muestra, 20\% en Dinamarca ${ }^{39}, 40 \%$ en Suecia ${ }^{40}$ y $46 \%$ en Israel ${ }^{41}$.

La amplia variación en la prevalencia del dolor observada en la literatura, incluido este trabajo, puede atribuirse a las diferencias en los criterios para definir dolor crónico (dolor que dura más de tres o seis meses) o a diferencias en la composición sociodemográfica de las muestras, por ejemplo, el género. Aunque en el presente estudio no se encontró diferencias significativas, hubo una mayor frecuencia en los hombres $(p=0,092)$ (Tabla 1), mientras que, en otros estudios, el dolor crónico es más frecuente en mujeres ${ }^{42}$.

La localización del dolor más frecuente fue cabeza / cara / boca (33,3\%) (Tabla 2). En comparación con un estudio realizado con pacientes con esquizofrenia en Sao Paulo ${ }^{23}$, nuestro estudio mostró una prevalencia menor de dolor lumbar. Al comparar la ubicación del dolor con la población general, nuestros hallazgos son similares a los del estudio de Kreling et al.$^{43}$ con respecto a las quejas de cabeza/cara/boca $(26,7 \%)$ y lumbar $(19,4 \%)$, primera y segunda localización más frecuente, respectivamente. En cuanto a la intensidad del dolor durante la última semana, el dolor más intenso e incapacitante fue reportado solo por $16,67 \%$ de pacientes (Tabla 2).

Este y otros estudios, demuestran que la prevalencia de síntomas dolorosos entre las personas con esquizofrenia es similar a la de la población general chilena, siendo de $32 \%{ }^{44}$. Sin embargo, se observan diferencias importantes en tres áreas. Primero los estudios experimentales muestran una elevación del umbral del dolor, segundo, los pacientes reportan su dolor con menor frecuencia y de manera más tardía, finalmente, existen barreras a la atención de salud para las personas con trastornos psicóticos severos, incluyendo, por ejemplo, la falta de una exploración dirigida de las manifestaciones del dolor ${ }^{45}$. La suma de estos factores contribuye inevitablemente a la mayor morbimortalidad de las personas con esquizofrenia ${ }^{46}$.

Este estudio presenta limitaciones, como el reducido tamaño de la muestra y las propias de un estudio de corte transversal, como la imposibilidad de establecer causalidad entre fenómenos. Sin embargo, entrega información a partir de una muestra local, respecto a la prevalencia y características del dolor clínico en un grupo de pacientes con esquizofrenia, siendo una queja poco atendida y que afecta su calidad de vida ${ }^{47}$. Al visibilizar esta temática se pretende contribuir a que el dolor sea considerado como una característica más a evaluar en personas con esquizofrenia, aumentando su búsqueda dirigida y su tratamiento oportuno.

\section{Referencias}

1. Loeser JD, Treede RD. The Kyoto protocol of IASP Basic Pain Terminology. Pain. 2008; 137 (3): 473-7. (1).

2. Price DD. Central neural mechanisms that interrelate sensory and affective dimensions of pain. Mol Interv. 2002; 2 (6): 392-402.

3. Harrison PJ. Neuropathology of schizophrenia. Psychiatry 2005; 4 (10): 18-21.

4. Sewards TV, Sewards MA. The medial pain system: Neural representations of the motivational aspect of pain. Brain Res Bull. 2002; 59 (3): 163-80.

5. Tracey I, Mantyh PW. The cerebral signature for pain perception and its modulation. Neuron. 2007; 55 (3): 377-91.

6. Valet M, Sprenger T, Boecker H, Willoch F, Rummeny E, Conrad B, et al. Distraction modulates connectivity of the cingulo-frontal cortex and the midbrain during pain-an fMRI analysis. Pain. 2004; 109 (3): 399-408.

7. Van Os J, Driessen G, Gunther N, Delespaul P. Neighbourhood variation in incidence of schizophrenia: Evidence for person-environment interaction. Br J Psychiatry 2000; 176 (MAR.): 243-8.

8. Andreasen NC. Schizophrenia: The fundamental questions. Brain Res Rev. 2000; 31 (2-3): 106-12.

9. Kraepelin E, Robertson, GM. Dementia Praecox and Paraphrenia. Edinburgh, Scotland: Livingstone 1919.

10. Bleuler E, Zinkin J, Lewis N. Dementia Praecox or The Group of Schizophrenias. New York, NY: International Universities Press. 1950.

11. Bonnot O, Anderson GM, Cohen D, Willer JC, Tordjman S. Are patients with schizophrenia insensitive to pain? A reconsideration of the question. Clin J Pain. 2009; 25 (3): 244-52.

12. Stubbs B, Thompson T, Acaster S, Vancampfort D, Gaughran F, Correll CU. Decreased pain sensitivity among people with schizophrenia: A meta-analysis of experimental pain induction studies. Pain. 2015; 156 (11): 2121-31.

13. Stubbs B, Mitchell AJ, De Hert M, Correll CU, Soundy A, Stroobants M, et al. The prevalence and moderators of clinical pain in people with schizophrenia: A systematic review and large scale meta-analysis. Schizophr Res. 2014; 160 (1-3): 1-8. 
14. Engels G, Francke AL, Van Meijel B, Douma JG, De Kam H, Wesselink W, et al. Clinical pain in schizophrenia: A systematic review. Vol. 15, Journal of Pain. Churchill Livingstone Inc. 2014: 457-67.

15. Potvin S, Marchand S. Hypoalgesia in schizophrenia is independent of antipsychotic drugs: A systematic quantitative review of experimental studies. Pain. 2008; 138 (1): 70-8.

16. Fishbain DA, Cutler RB, Lewis J, Cole B, Rosomoff RS, Rosomoff HL. Do the second-generation "atypical neuroleptics" have analgesic properties? A structured evidence-based review. Pain Med. 2004; 5: 359-65.

17. Kuritzky A, Mazeh D, Levi A. Headache in schizophrenic patients: a controlled study. Cephalalgia. 1999; 19 (8): 725-7.

18. Leucht S, Burkard T, Henderson J, Maj M, Sartorius N. Physical illness and schizophrenia: A review of the literature. Acta Psychiatr Scand. 2007; 116 (5): 317-33.

19. De Almeida JG, Braga PE, Neto FL, Pimenta CA de M. Chronic pain and quality of life in schizophrenic patients. Rev Bras Psiquiatr. 2013; 35 (1): 13-20.

20. Mahmood T, Romans S, Silverstone T. Prevalence of migraine in bipolar disorder. J Affect Disord. 1999; 52 (1-3): 239-41.

21. McIntyre RS, Konarski JZ, Wilkins K, Bouffard B, Soczynska JK, Kennedy SH. The prevalence and impact of migraine headache in bipolar disorder: Results from the Canadian Community Health Survey. Headache. 2006; 46 (6): 973-82.

22. Miranda JP, Quezada P, Caballero P, Jiménez L, Morales A, Bilbeny N, et al. Revisión Sistemática: Epidemiología de Dolor Crónico No Oncológico en Chile. Rev El Dolor 2013; 59: 10-7.

23. De Almeida JG, Kurita GP, Braga PE, Pimenta CA de M. dor crônica em pacientes esquizofrênicos: Prevalência e características. Cad Saude Publica 2010; 26 (3): 591-602.

24. Melzack R. The McGill Pain Questionnaire: Major properties and scoring methods. Pain. 1975; 1 (3): 277-99.

25. Waldman S. Pain Assessment Tools for Adults. Segunda Edición. In Pain Review. Editorial Elsevier 2009; 222.

26. World Health Organization. The ICD-10 classification of mental and behavioral disorders: clinical description and diagnostic guidelines. Geneva, 1992. https://www. who.int/classifications/icd/en/bluebook.pdf (accessed 22 January 2020).

27. Melzack R. Pain - An overview. Acta Anaesthesiol Scand. 1999; 43 (9): 880-4.

28. Lorenz J, Minoshima S, Casey K. Keeping pain out of mind: the role of the dorsolateral prefrontal cortex in pain modulation. Brain 2003; 126 (5): 1079-91.

29. Vogt BA, Sikes RW. The medial pain system, cingulate cortex, and parallel processing of nociceptive information. Prog Brain Res. 2000; 122: 223-35.

30. Cole MW, Anticevic A, Repovs G, Barch D. Variable global dysconnectivity and individual differences in schizophrenia. Biol Psychiatry 2011; 70: 43-50.

31. Goldman LS. Medical illness in patients with schizophrenia. J Clin Psychiatry. 1999; 60 Suppl. 21: 10-5.

32. Kritharides L, Chow V, Lambert TJ. Cardiovascular disease in patients with schizophrenia. Med J Aust. 2017; 206 (2): 91-5.

33. Laursen TM, Nordentoft M. He@art disease treatment and mortality in schizophrenia and bipolar disorder changes in the Danish population between 1994 and 2006. J Psychiatr Res. 2011; 45 (1): 29-35.

34. Casey DA, Rodríguez M, Northcott C, Vickar G, Shihabuddin L. Schizophrenia: medical illness, mortality, and aging. Int J Psychiatry Med. 2011; 41 (3): 245-51.

35. Ishikawa $H$, Yasunaga $H$, Matsui $H$, Fushimi K, Kawakami N. Differences in cancer stage, treatment, and in-hospital mortality between patients with and without schizophrenia: retrospective matched-pair cohort study. Br J Psychiatry 2016; 208 (3): 239-44.

36. Watson GD, Chandarana PC, Merskey H. Relationships between pain and schizophrenia. Br J Psychiatry 1981; 138 (1): 33-6.

37. Chaturvedi SK, Michael A. Chronic pain in a psychiatric clinic. J Psychosom Res. 1986; 30 (3): 347-54.

38. Smith BH, Elliott AM, Alastair Chambers W, Smith WC, Hannaford PC, Penny K. The impact of chronic pain in the community. Fam Pract. 2001; 18 (3): 292-9.

39. Sjøgren P, Ekholm O, Peuckmann V, Grønbæk M. Epidemiology of chronic pain in Denmark: An update. Eur J Pain. 2009; 13 (3): 287-92.

40. Brattberg G, Thorslund M, Wikman A. The prevalence of pain in a general population. The results of a postal survey in a county of Sweden. Pain. 1989; 37 (2): 21522.

41. Neville A, Peleg R, Singer Y, Sherf M, Shvartzman P. Chronic pain: A population-based study. Isr Med Assoc J. 2008; 10 (10): 676-80.

42. Mathias SD, Kuppermann M, Liberman RF, Lipschutz RC, Steege JF. Chronic pelvic pain: Prevalence, health-related quality of life, and economic correlates. Obstet Gynecol. 1996; 87 (3): 321-7.

43. Kreling MCGD, da Cruz DALM, Pimenta CA de M. Prevalência de dor crônica em adultos. Rev Bras Enferm. 2006; 59 (4): 509-13.

44. ACHED-CP, 2019. Recomendaciones nacionales para el diagnóstico, prevención, el tratamiento del dolor crónico en pacientes adultos. Consenso chileno de expertos.

45. Angermeyer MC, Matschinger $\mathrm{H}$. The stigma of mental 
illness: efects of labelling on public attitudes towards people with mental disorder. Acta Psychiatr Scand 2003: 108: 304-9.

46. Muir- Cochrane E. Medical co-morbidity risk factors and barriers to care. J Psychiatr Ment Health Nurs.
2006; 13 (2003): 447-52.

47. Van Hasselt FM, Schorr SG, Mookhoek EJ, Brouwers JRBJ, Loonen AJM, Taxis K. Gaps in health care for the somatic health of outpatients with severe mental illness. Int J Ment Health Nurs. 2013; 22 (3): 249-55. 